\title{
Simulation of electrostatic properties and gas amplification in microstrip gas chambers and comparison with measurements *
}

\author{
S. Schmidt, U. Werthenbach and G. Zech \\ Universität Siegen
}

December 15, 1993

\begin{abstract}
The electrostatic field in MSGCs is computed using the charge simulation method. Bulk and surface currents are taken into account as well as dielectric properties. The gas amplification and the electric field are estimated as a function of the geometric parameters of the structure, of the applied voltages, and of the conductivity of the substrate. The simulations are compared with measurements using glass substrates of 50,100 and $500 \mu m$ thickness. They show that for a pad readout at the back plane thin substrates and large gaps between anode and cathode strips are favorable. The asymmetry of the signals induced on the cathode strips should allow to improve the position resolution of MSGCs.
\end{abstract}

*Work supported by Bundesminister für Forschung und Technologie (FK 056Si79I) 


\section{Introduction}

Gas microstrip detectors in which the wires of the standard multiwire proportional chambers are replaced by lithographically produced strips on solid substrates have had many interesting developments since the first realization by A. Oed [1]. References to most of the published results can be found in $[2,3,4]$.

The production and test of different structures is a tedious and expensive procedure which could be reduced if reliable simulations with predictive power were available. Very encouraging steps in this direction have been done $[5,6,7,8]$. The investigations of ref. [5] are similar to those presented here, but differ in the assumptions made for the substrate properties.

In [5] and [8] the electric field is calculated with a multigrid technique. We have applied the charge simulation method and included the influence of surface and bulk currents. To allow comparisons with experimental data we have also simulated the gas amplification for the standard 90:10 argon methane mixture.

In the following section we describe the method, which then is used in section 3 to compute the field for different configurations and compare the simulation to measurements with glass substrates. Finally in section 4 we estimate the effect of some geometric parameters on the field, the gas amplification, the anode capacitance, and the size of signals induced on the cathode strips and back plane electrodes.

\section{The simulation model}

The processes occurring in a MSGC are far too complicated to be simulated in detail. We have avoided the introduction of adjustable empirical parameters to account for these processes and preferred to use a simple model. As a consequence the computations are not necessarily valid for all operating conditions of the detector and may limit the applicability of the results.

For the computation of the electric field we neglect the ions in the gas. Thus conditions, where the performance is deteriorating due to high rates are not simulated.

\subsection{Substrate properties}

Glasses and plastic substrates have a non negligible surface conductivity which depends critically on the treatment of the surface [9].

Experimentally it is known that the conductivity in glasses increases with the field strength [10]. We neglect this field dependence and assume uniform bulk and surface resistivities.

A possible charge carrier depletion zone near the anode, which could be caused by the migration of the alkali ions in glasses [11] is also ignored. The high resistivity in this region would be accompanied by a high field and increased gas amplification [12]. So far we have not observed this effect under normal conditions with the glass (Schott D263) used by us. However in long term applications a rising gain is expected. To verify this prediction we have reduced the resistivity of the substrate by more than two orders of magnitude by heating it to $60^{\circ} \mathrm{C}$ for two days. After cooling down the gain had raised by about $30 \%$, when the high voltage was on during heating, but remained 
unchanged when the glass was heated with the voltage switched off. The depletion could be reversed by changing the polarity of the electric field (see figure 1). The integrated current during heating corresponds to one year of operation at room temperature. Thus most measurements with borosilicate glasses should be free of depletion effects. Therefore we neglected them in our simulations.

The dielectric constant of the substrate is assumed to be independent of the electric field.

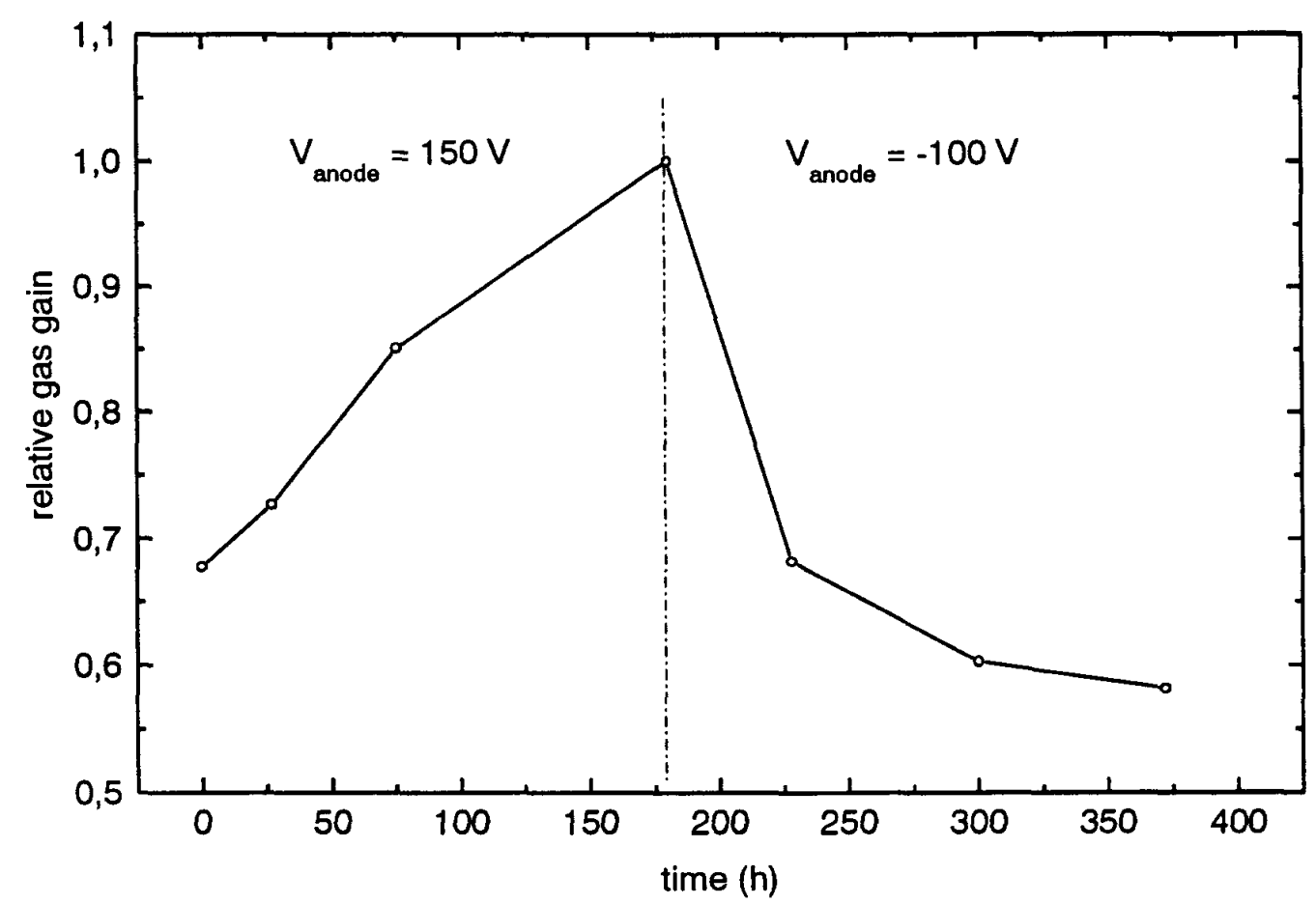

Figure 1: Gas amplification as a function of time. The substrate was heated to $60^{\circ} \mathrm{C}$ with positive anode voltage. The anode voltage was reversed after 180 hours.

\subsection{Electrodynamic treatment}

In the stationary case the electric displacement and the electric current density outside the electrodes follow analogous equations:

$$
\begin{array}{rlrl}
\nabla \cdot \mathbf{D} & =0 ; & \mathbf{D}=\epsilon \epsilon_{0} \mathbf{E} \\
\nabla \cdot \mathbf{j}=0 ; & \mathbf{j}=\sigma \mathbf{E}
\end{array}
$$

The continuity equations for the electric current density and the electric displacement lead in both cases to a discontinuity of the electric field at boundaries of different media. 
The change of the field component perpendicular to the surface is generated by surface charges.

The equations (1) and (2) cannot be satisfied simultaneously. For fields varying with time constants much shorter than $\tau=\rho \epsilon \epsilon_{0}$, where $\rho$ and $\epsilon$ are the specific resistivity and the dielectric constant of the substrate, currents can be neglected, whereas for time constants much larger than $\tau$ the dielectric properties are unimportant.

Typical values for $\tau$ lie between $10^{2} \mathrm{~s}$ and $10^{4} \mathrm{~s}$ for the usual substrates used in MSGCs. When the voltages of MSGC are switched on, first polarization effects dominate and determine the field. Currents start to flow and to charge up the surface of the substrate. Simultaneously the field at the anode and the gain decrease with time constants ranging from a fraction of a second to hours, depending on the resistivity of the substrate. For a standard well conducting glass stable conditions will be reached after a few minutes. Then the potentials and the field will be defined only by the resistivities. (The situation is similar to that for two serial capacitors when a constant voltage is switched on. Initially the potential between the capacitors is defined by their capacitance, but due to leakage currents it will settle after some time to the value corresponding to the resistivities.)

In ref. [13] the initial decrease in gain is explained by the depletion of alkali ions near the anode. However this effect would increase the gain. Also a slow polarization of the substrate as proposed in ref.[2] would not lead to the observed gain drop, however a small electric surface conductivity, which is unavoidable, probably fully accounts for it.

Usually the stationary case (2) is of interest, but for the computation of induced signals the time constants are of the order of nanoseconds to microseconds. Then only the dielectric properties of the substrate have to be considered and equation (1) will be used. The surface currents will not influence the induced signals or the relevant capacitance, even for substrates with surface resistivities below $10^{10} \Omega \mathrm{cm}$.

\subsection{Computation of the electric field}

For the numerical computation of the electric field we have extended an existing program [14] based on the method of image charges in two dimensions. The unknown charge strength of a certain number of charge elements are computed through the requirement, that the superposition of their potentials agree with the applied voltages. This leads to a set of linear equations which can be solved by matrix inversion.

To model the MSGC we only use two types of charge elements: uniformly charged strips and isolated strips. An isolated strip creates an electric field which is infinite at the two edges. The charge distribution of electrodes is simulated by a superposition of one isolated strip and several uniformly charged substrips. The isolated strip reproduces the strong field at the edges. Thus no fine subdivision is necessary. The electrodes are assumed to have negligible thickness.

Uniformly charged strips are also used in the program to simulate the surface charges which are either due to polarization or to currents.

Surface currents are simulated by introducing a thin layer of thickness $d_{s}$, typically $1 \mu m$, of high bulk conductivity $\sigma_{1}$. The corresponding surface conductivity is then $\sigma_{s}=\sigma_{1} d_{s}$. Only the ratio $R_{\sigma}=\sigma_{s} / \sigma_{v}$ where $\sigma_{v}$ is the bulk conductivity is relevant for the static properties of a MSGC. (In technical applications the surface conductivity 
is defined differently. There it is obtained from a current measurement which is also sensitive to the bulk conductivity and therefore to the thickness of the substrate.)

We profit from the symmetry of the MSGCs to reduce the number of independent charge elements. Reflection symmetry and translation symmetry are applied. However some strips at the borders of the device are allowed to have charge distributions different from the central ones. This allows us to study border effects.

To get a sufficiently precise result the gap between the anode and the potential strips has to be filled by a large number of surface charges. We chose strips of variable widths, ranging from $1 \mu \mathrm{m}$ to $3 \mu \mathrm{m}$ (see figure 2).

Typically we use some $10^{4}$ charge elements, of which about 300 to 500 are independent. The computation is relatively fast and precise. The program can be run on 'personal computers' but when many field lines have to be computed it is advisable to use faster computers.

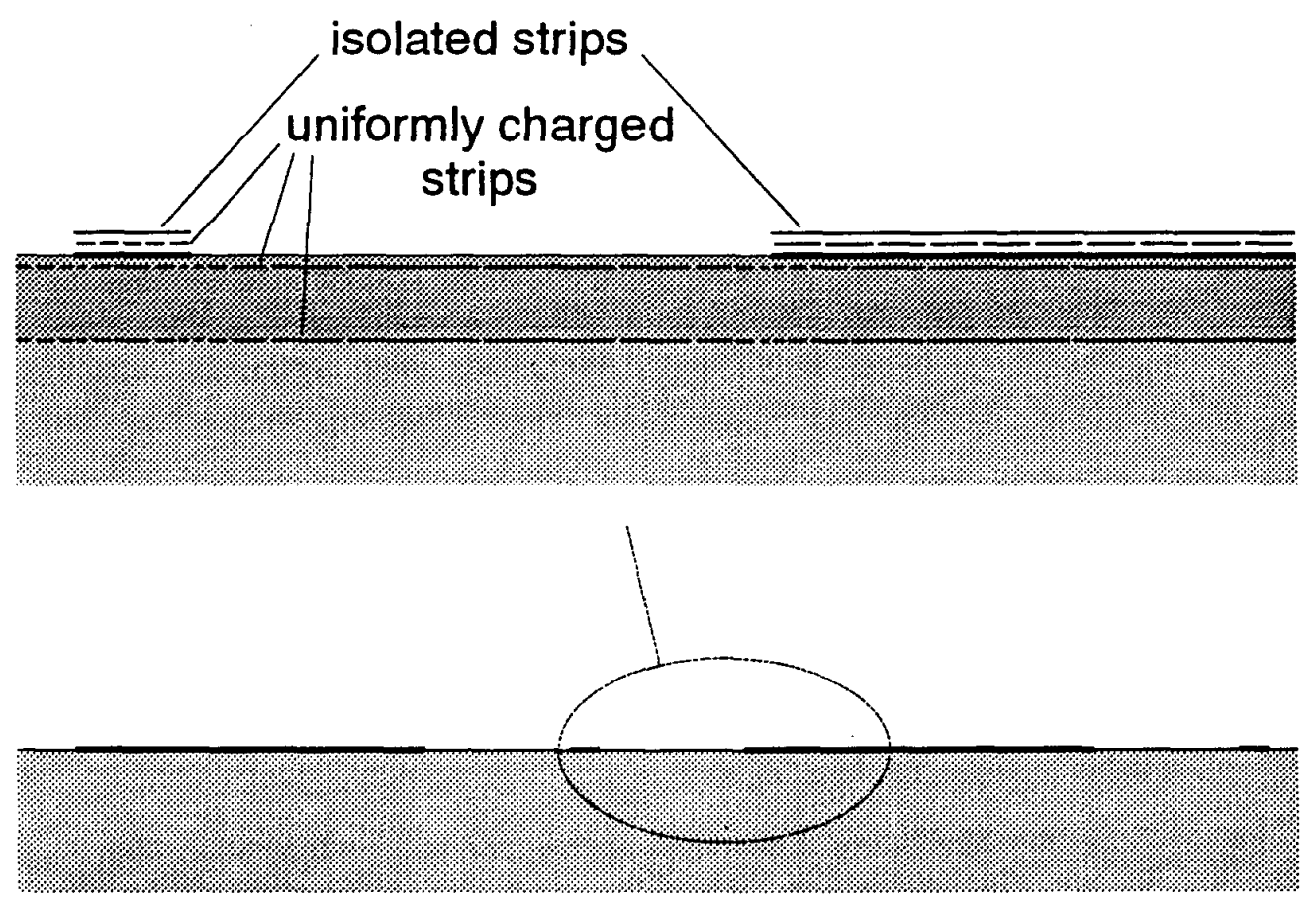

Figure 2: Schematic sketch (not to scale) of the MSGC configuration used for the simulation. The blow-up shows the charge elements used in the simulation of the electrodes and the surface charges at the boundary of the substrate and below the high $\sigma$ layer.

\subsection{Gas amplification}

The gas amplification was computed from the simple approximation [15]

$$
\alpha / p=A \exp \frac{-B}{(E / p)^{0.5}}
$$




\begin{tabular}{|l|r|r|r|r|r|}
\hline & model & \multicolumn{2}{|c|}{ E-field $(\mathrm{kV} / \mathrm{mm})$} & \multicolumn{2}{|c|}{ gain } \\
& & $\mathrm{d}=500 \mu \mathrm{m}$ & $\mathrm{d}=100 \mu \mathrm{m}$ & $\mathrm{d}=500 \mu \mathrm{m}$ & $\mathrm{d}=100 \mu \mathrm{m}$ \\
\hline a) & vacuum & 32.7 & 35.6 & $10.510^{3}$ & $18.310^{3}$ \\
b) & dielectric & 32.5 & 36.9 & $10.110^{3}$ & $23.310^{3}$ \\
c) & $\sigma_{v} \neq 0, \sigma_{s}=0$ & 32.4 & 37.4 & $9.910^{3}$ & $25.910^{3}$ \\
d) & $\sigma_{v}=0, \sigma_{s} \neq 0$ & 11.8 & 11.9 & $0.510^{3}$ & $0.510^{3}$ \\
e) & $\sigma_{s} / \sigma_{v}=0.004 \mathrm{~cm}$ & 15.5 & 17.3 & $1.310^{3}$ & $2.510^{3}$ \\
f) & $\sigma_{s} / \sigma_{v}=0.004 \mathrm{~cm}, V_{B}=-300 \mathrm{~V}$ & 16.2 & 20.6 & $1.710^{3}$ & $8.010^{3}$ \\
\hline
\end{tabular}

Table 1: Field strength and gas amplification for different models and substrate thicknesses. The values are given for the center of the anode. The first column corresponds to the indices in figure 3 and 4 .

where $\alpha$ is the first Townsend coefficient, p the pressure, and A, B constants depending on the gas properties.

For the standard gas mixture of argon methane $90: 10$, to which we refer throughout this paper, we use [15] $\mathrm{A}=5.1 \mathrm{~mm}^{-1}$ torr $^{-1}$ and $\mathrm{B}=3.2 \cdot 10^{2} \mathrm{~V}^{0.5} \mathrm{~m}^{-0.5}$ torr $^{-0.5}$. The constants are valid for room temperature $\left(20^{\circ} \mathrm{C}\right)$. (In principle the scaling with the pressure in the above equation should be replaced by scaling with the density [16]).

The calculated gas amplification for a drift tube was checked against a measurement with charge sensitive amplifier with long integration time. Agreement within $20 \%$ was found. However the parametrization of [15] differs strongly from other published results [17].

We believe that the uncertainty in the normalization of the computed gas amplification is of the order of $30 \%$. The relative uncertainty comparing gas amplifications for different parameters of MSGCs is much smaller.

\section{Results}

\subsection{Electric field in MSGCs}

Most of the following numerical estimates correspond to a configuration with $8 \mu m$ anode width, $200 \mu \mathrm{m}$ wide cathode strips and a gap of $120 \mu \mathrm{m}$ between the electrodes. The voltage applied to the anode and the drift electrode are $V_{A}=630 \mathrm{~V}$ and $V_{D}=-3000 \mathrm{~V}$, respectively. The distance of the drift electrode from the substrate is $10 \mathrm{~mm}$. The back plane is grounded. Figure 3 and 4 shows field calculations for $500 \mu \mathrm{m}$ and $100 \mu \mathrm{m}$ thick plates. The electric fields at the center of the anodes and gas amplifications are given in table 1.

In figure 3a the field has been calculated naively, neglecting the conductivity and the dielectricity of the substrate.

In figure $3 \mathrm{~b}$ a dielectric constant of $\epsilon=5$ is assumed. The field corresponds to the situation immediately after the voltages have been switched on $(t \ll \tau)$.

The field for a conducting substrate, but neglecting surface currents is shown in fig. $3 c$ for the stationary case $(t \gg \tau)$. 
When the thickness of the substrate is reduced to $100 \mu \mathrm{m}$ the field as shown in figures $4 \mathrm{a}$ to $4 \mathrm{c}$ is still very similar in the three cases, but differs considerably from that of the thicker substrate. Now the grounded back plane attracts the field lines leaving the anode. Such a configuration would lead to deposition of positive ions at the surface and would not allow a stable operation.

In figure $3 \mathrm{~d}$ and $4 \mathrm{~d}$ the surface of the substrate is conducting, but the bulk resistivity is infinite. The potential in the gap between anode and cathode strips now decreases linearly with the distance from the anode. The thickness of the substrate and the potential applied to the back plane are irrelevant in this situation. It is obvious that the surface conductivity has a large effect on the field (see table 1). The field strength at the anode center drops by about a factor three compared to the previous situation.

The field maps for a realistic situation, where both surface and bulk currents flow are displayed in figures $3 \mathrm{e}$ and $4 \mathrm{e}$. The selected ratio of the conductivities is $R_{\sigma}=4 \cdot 10^{-3} \mathrm{~cm}$, which is a typical value for a glass with good ionic conductivity. The drift lines now cover almost the entire anode.

In summary, surface currents have two important consequences: They reduce drastically the field strength and the gain and they push the field lines leaving the anode away from the substrate surface and thus reduce a possible deposition of positive ions. However, for very thin foils and large gaps between anode and cathode strips surface currents will not be sufficient to avoid charge-up completely. The initial gain drop usually observed when the high voltage is applied to a MSGC can be explained by the setting in of surface currents. When surface currents are absent, the surface is charged up by bulk currents only. In this case the gain for thin substrates would slightly increase [5] as can be seen by comparing cases b) and c) in table 1 .

Figure 5 shows the surface charge between anode and cathode. In the absence of surface conductivity the charge is very small, especially for thick substrates. Even small surface conductivities lead to large charge densities near the borders of the strips which explain the decrease in gain.

The strong variation of the gain with the ratio $R_{\sigma}$ leads to a rate dependent gas amplification for substrates where $R_{\sigma}$ is radiation sensitive $[18,4]$. The observed rate dependence $[13,12]$ should not be attributed exclusively to ion deposition.

Finally figures $3 \mathrm{f}$ and $4 \mathrm{f}$ show the electric field for conditions similar to figures $3 \mathrm{e}$, $4 e$ but for a back plane potential of $-300 \mathrm{~V}$. As expected the field at the anode and the gain are increased, the effect being smaller for the thicker plate. Qualitatively the shape of the field map is not affected drastically. Positive back plane voltages as proposed in ref. $[1,13]$ to avoid charging up, according to our calculations, do not seem to improve the performance of MSGCs.

\subsection{Comparison with measurements}

From the results of the previous section we learn that the ratio of the surface and bulk conductivities $R_{\sigma}=\sigma_{s} / \sigma_{v}$ is a crucial parameter for the gain of a MSGC. The surface conductivity depends not only on the substrate material, but also on the treatment it has undergone. Etching, cleaning, heating, deposition of ions and radiation may influence it. Thus, $\sigma_{s}$ is hard to predict. For our comparison we adjust $R_{\sigma}$ to the data. Most of the measurements used for the comparison are described in [4]. In addition we have 


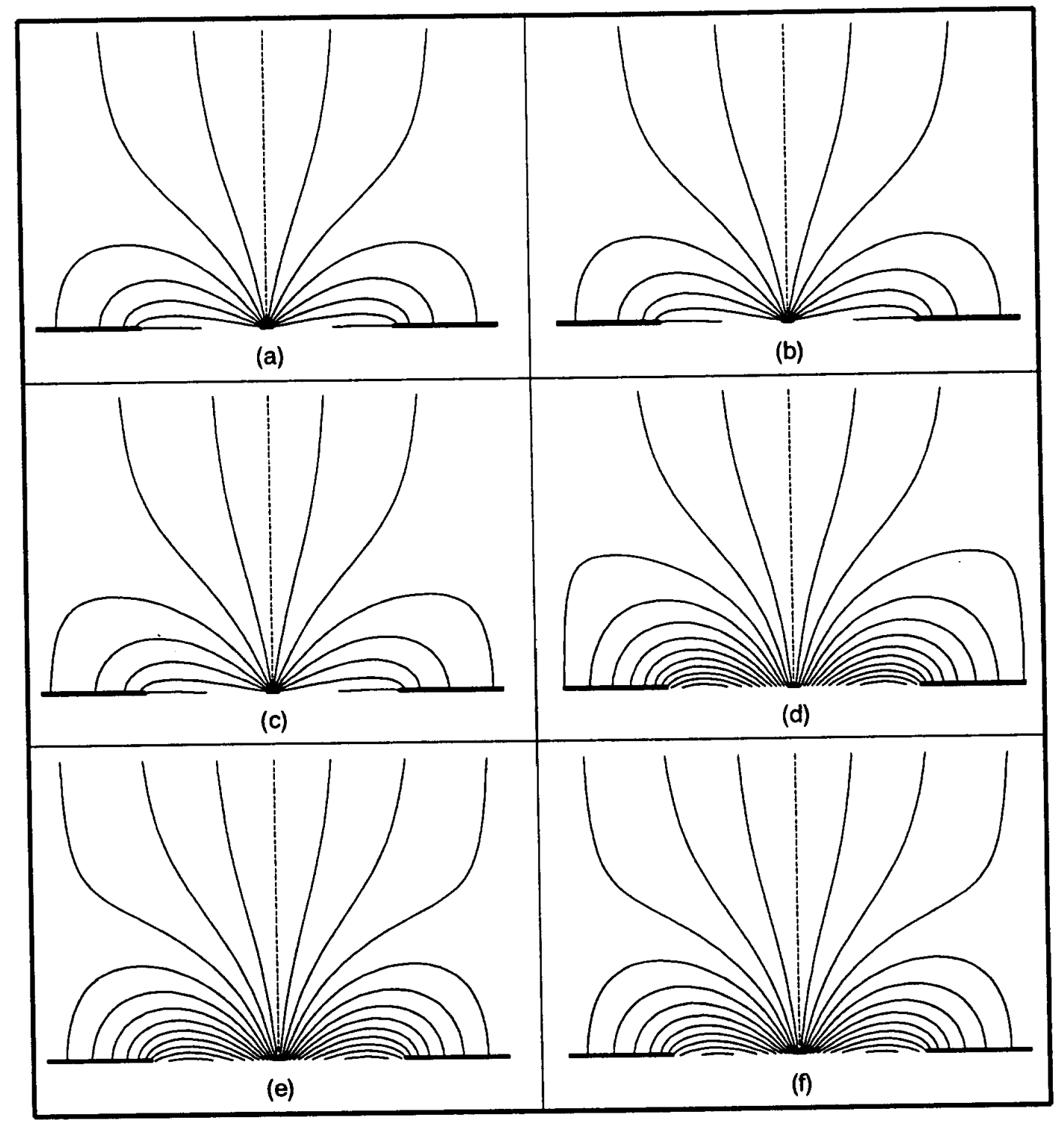

Figure 3: Field maps for a $500 \mu m$ thick glass substrate. a) $\epsilon=1, \sigma=0, b) \epsilon=5, \sigma=0$, c) $\left.\left.\left.\sigma_{v} \neq 0, \sigma_{s}=0, d\right) \sigma_{v}=0, \sigma_{s} \neq 0, e\right) \sigma_{s} / \sigma_{v}=4 \cdot 10^{-3} \mathrm{~cm}, f\right) \sigma_{s} / \sigma_{v}=4 \cdot 10^{-3} \mathrm{~cm}$ and $V_{B}=-300 \mathrm{~V}$. 


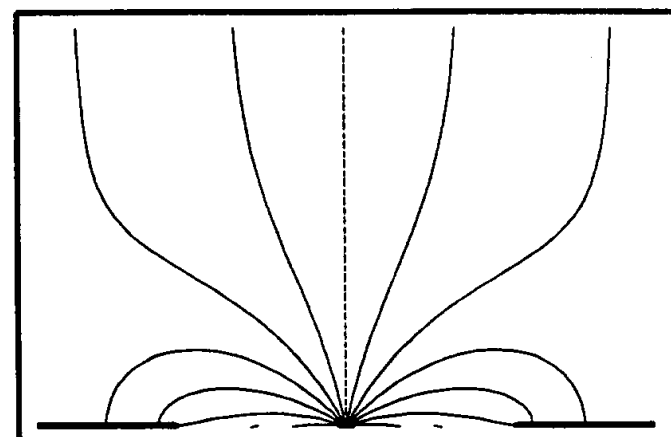

(a)

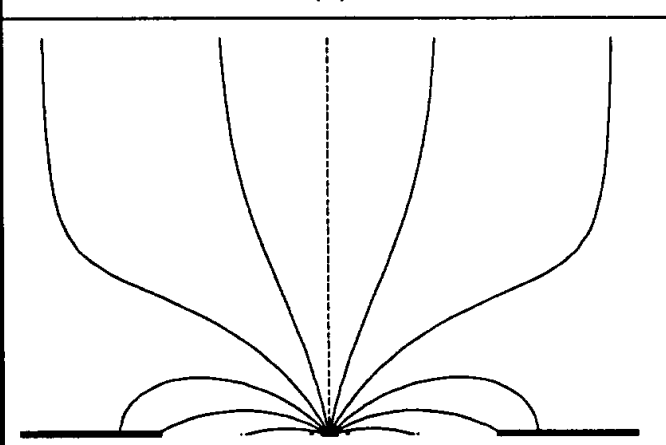

(c)

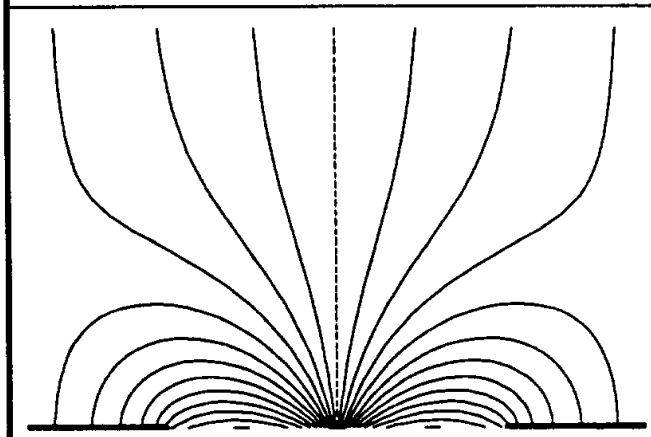

(e)

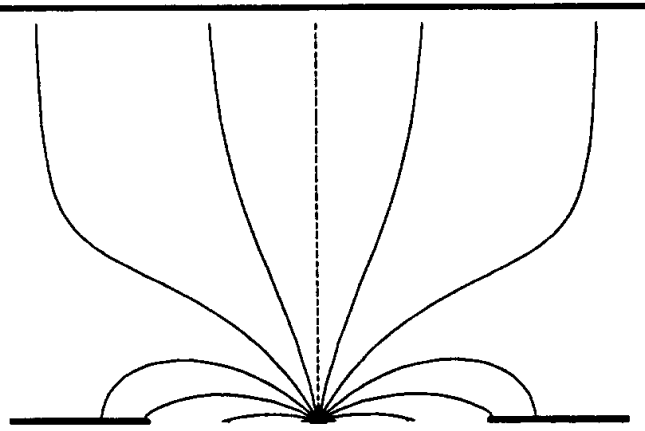

(b)

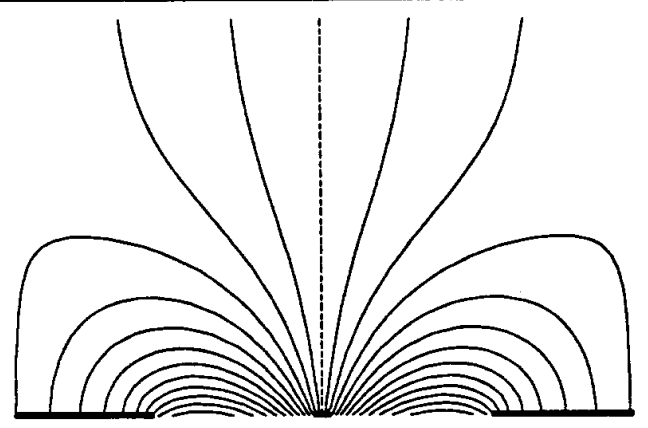

(d)

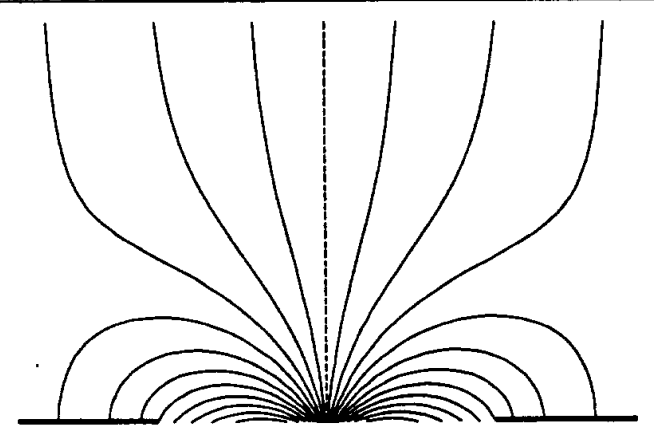

(f)

Figure 4: Field maps for a $100 \mu \mathrm{m}$ thick glass substrate. a) $\epsilon=1, \sigma=0, b) \epsilon=5, \sigma=0$, c) $\left.\left.\left.\sigma_{v} \neq 0, \sigma_{s}=0, d\right) \sigma_{v}=0, \sigma_{s} \neq 0, e\right) \sigma_{s} / \sigma_{v}=4 \cdot 10^{-3} \mathrm{~cm}, f\right) \sigma_{s} / \sigma_{v}=4 \cdot 10^{-3} \mathrm{~cm}$ and $V_{B}=-300 \mathrm{~V}$. 


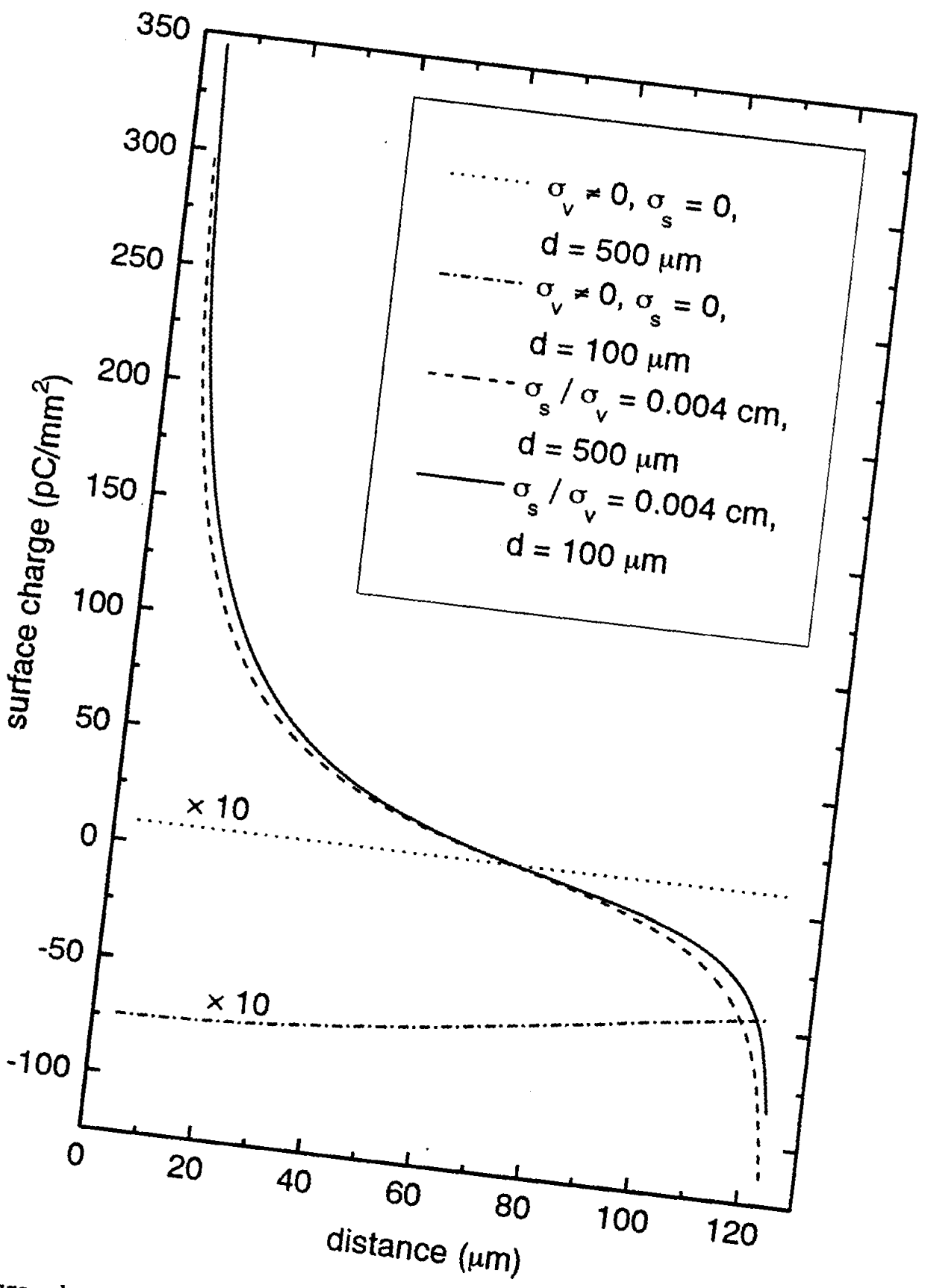
center for different surface and buik conductivities. The of the distance to the anode
conductivity are multiplied by a factor of ten. 
tested MSGCs on $50 \mu m$ thin glass foils. The back plane was floating and the foil was suspended in the gas. The behavior of this thin foil is very similar to that of the other plates.

Figure 6a shows the gain as a function of the anode voltage for a $0.5 \mathrm{~mm}$ thick glass plate and parameters as described in section 3.1 (except for a larger anode of $10 \mu \mathrm{m}$ width) and the predictions for different ratios $R_{\sigma}$. Good agreement is found for $R_{\sigma}=$ $0.6 \cdot 10^{-2}$. The gain for the $100 \mu \mathrm{m}$ and $50 \mu \mathrm{m}$ thin substrates, as shown in figure $6 \mathrm{~b}$ and $6 \mathrm{c}$ are about 30 percent lower and can be described by a ratio $R_{\sigma}=2 \cdot 10^{-2}$. The surface conductivities for thin glass seems to be higher than for thick glass. Differences in the conductivities might be explained by different cooling speeds in the production process.

The gain dependence on the drift voltage is compared to the calculation in figure 7 . Again the simulation provides a fairly good description of the measurements.

In figure 8 , finally we varied the back plane voltage. The simulation is able to qualitatively account for the rise of the gain with the decrease of $V_{b}$ but the prediction is steeper than the measurement. We have not found a convincing explanation for this effect. Possibly the stronger electric field enhances the bulk conductivity relative to the surface conductivity. However, the expected effect [11] is about a factor three smaller than the observed one.

\section{Predictions}

\subsection{Gain dependence on strip pattern and conductivity}

First we investigate the gas amplification as a function of the position of the avalanche at the anode.

Substrates of thicknesses of $500 \mu m$ were considered with the cell parameters $w_{A}=$ $10 \mu m, w_{G}=120 \mu m$ and $w_{C}=200 \mu m$.

Figure 9 gives the electric field at $1 \mu \mathrm{m}$ above the surface of the anode as a function of the lateral distance to the center of the anode for different ratios of the surface current to the bulk current. For low surface conductivities the field variations are rather large and seem to indicate that also the gas amplification would vary drastically. Our computations (figure 9) show that this is not the case. The gain variations are rather moderate. The good energy resolution of MSGCs was originally attributed to a field configuration where all drift lines emerging from the cathode plane would end in a very small region in the center of the anode strip [21].

In MSGCs gas amplifications of about $10^{3}$ to $10^{4}$ are obtained, which is low compared to wire chambers. One factor limiting the gain is field emission from the cathode strips. There are possibilities to de-activate the edges of the strips by covering them by a thin insulating layer [19]. This technique is not yet well tested and adds another step to the production process of the structures. Thus one should try to maximize the gain by an optimum selection of the strip pattern.

In figure $10 \mathrm{a})$ to $c$ ) we present the gain and the electric fields near ( $1 \mu \mathrm{m}$ above) the edges of the cathode strip and the anode strip as a function of three parameters, namely anode width $w_{A}$, cathode width $w_{C}$ and gap width $w_{G}$.

As expected a small anode width is favorable. Less obvious is the strong dependence 


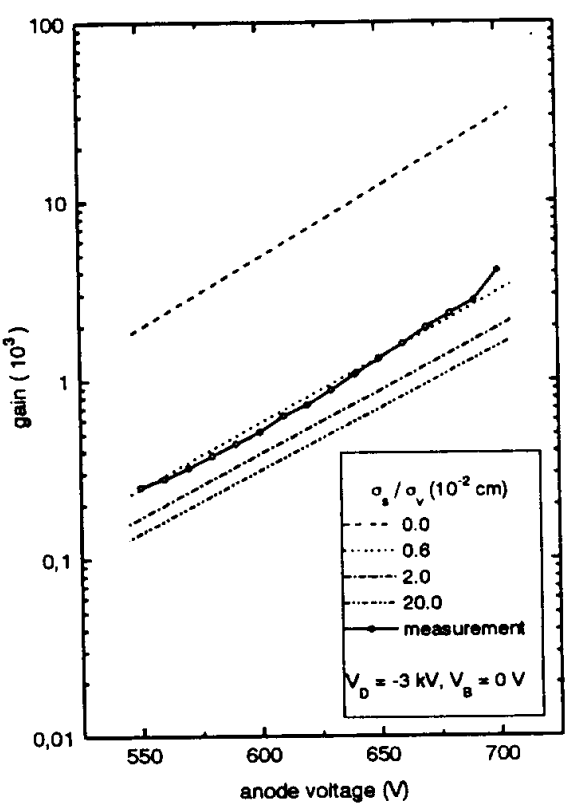

(a)

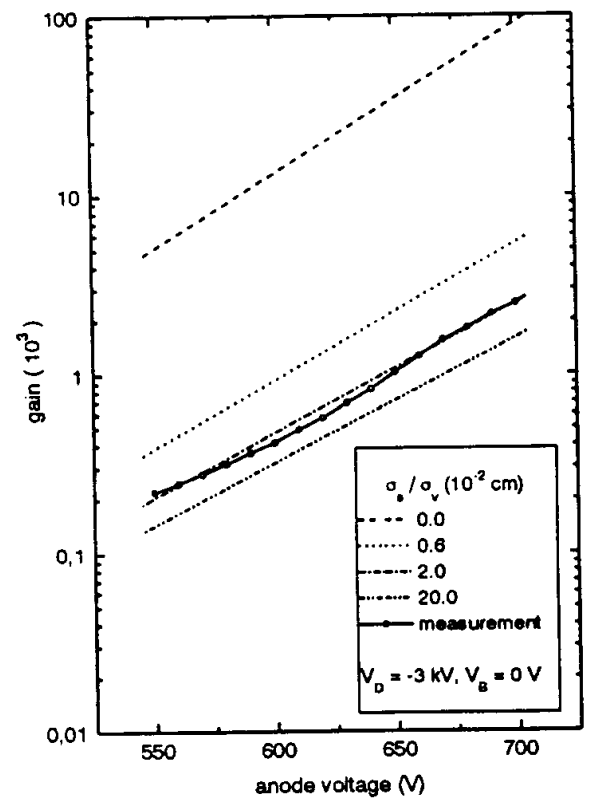

(b)

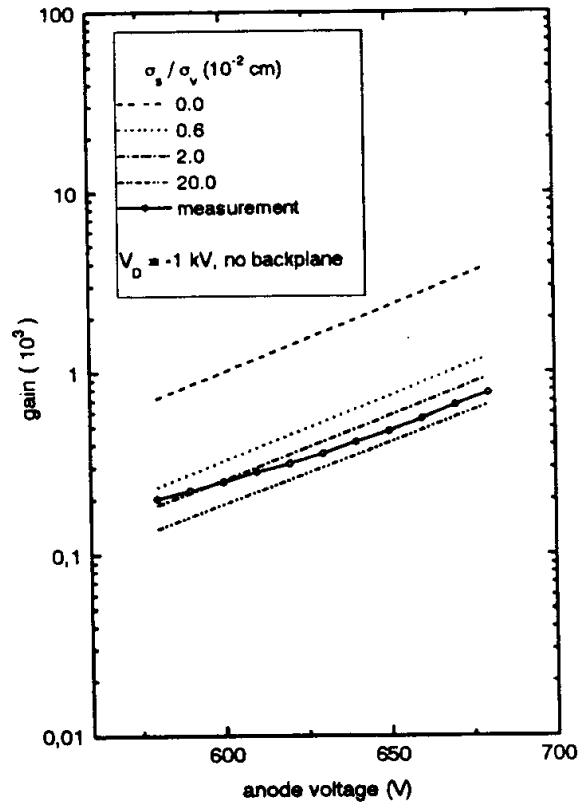

(c)

Figure 6: Gas amplification for 500 (a), 100 (b) and $50 \mu \mathrm{m}$ (c) glass plates as a function of the anode voltage. 

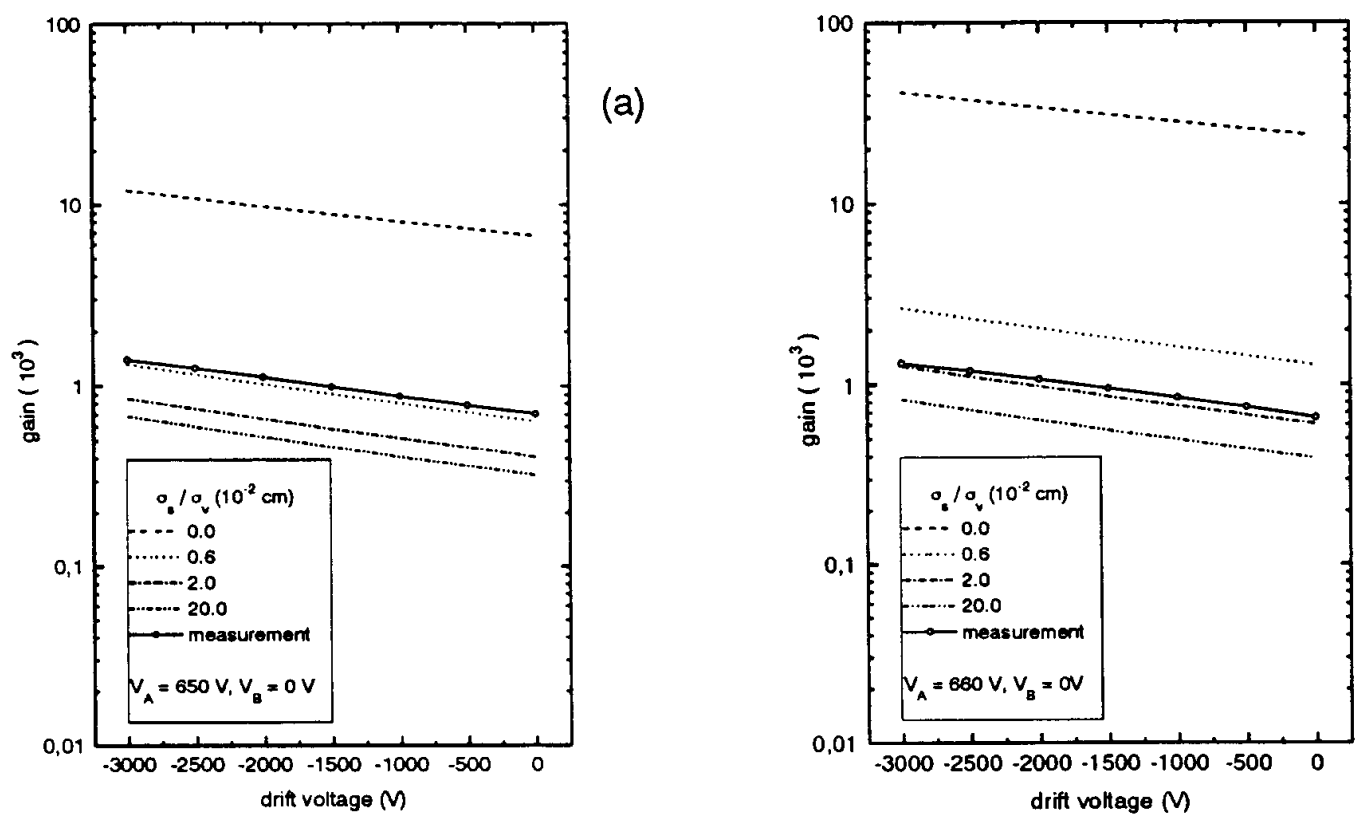

(b)

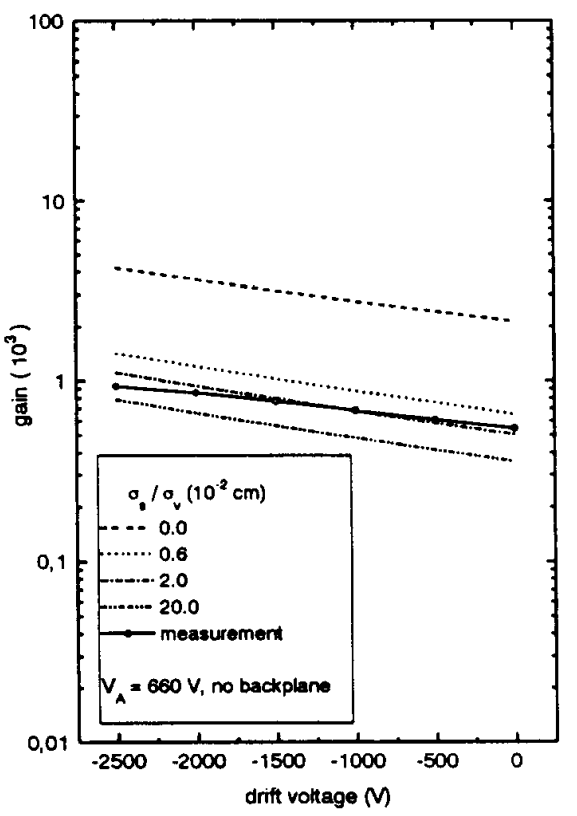

(c)

Figure 7: Gas amplification for $500(a), 100(b)$ and $50 \mu \mathrm{m}$ (c) glass plates as a function of the drift voltage. 


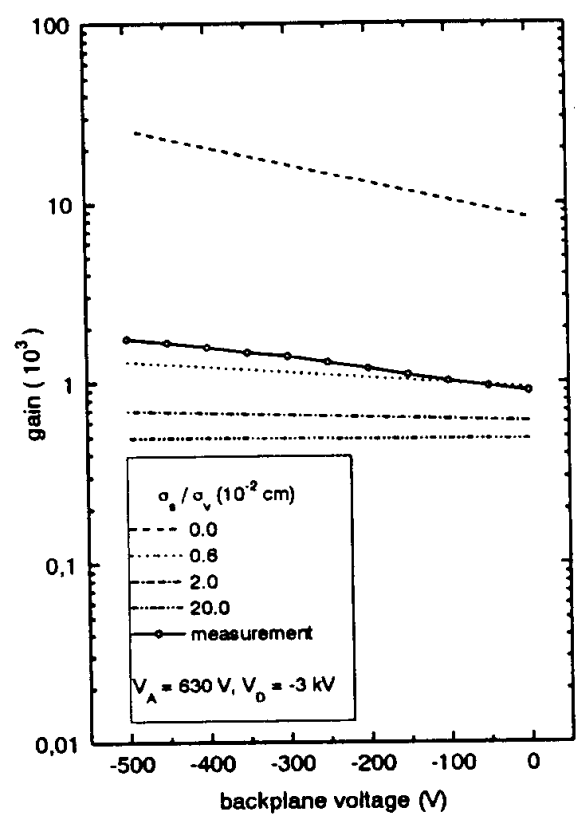

(a)

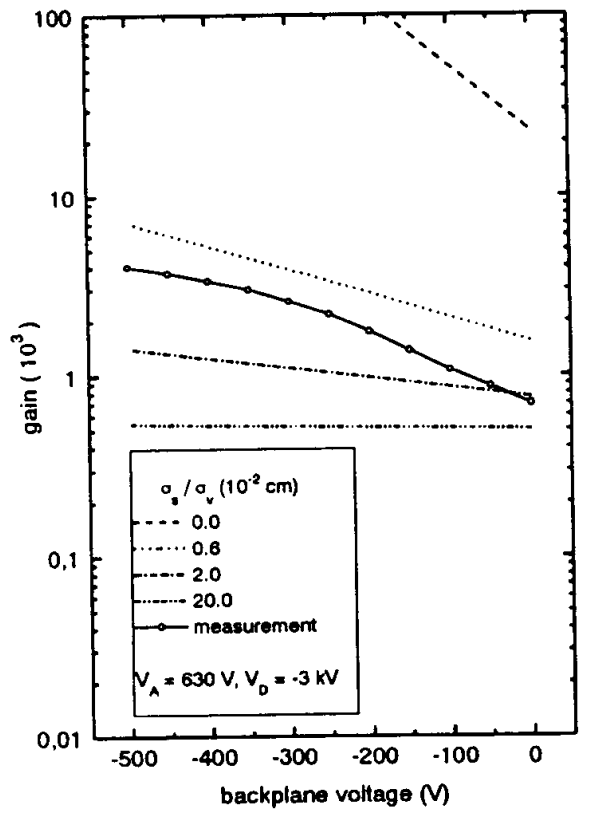

(b)

Figure 8: Gas amplification for 500 (a) and $100 \mu \mathrm{m}$ (b) glass plates as a function of the back plane voltage. 


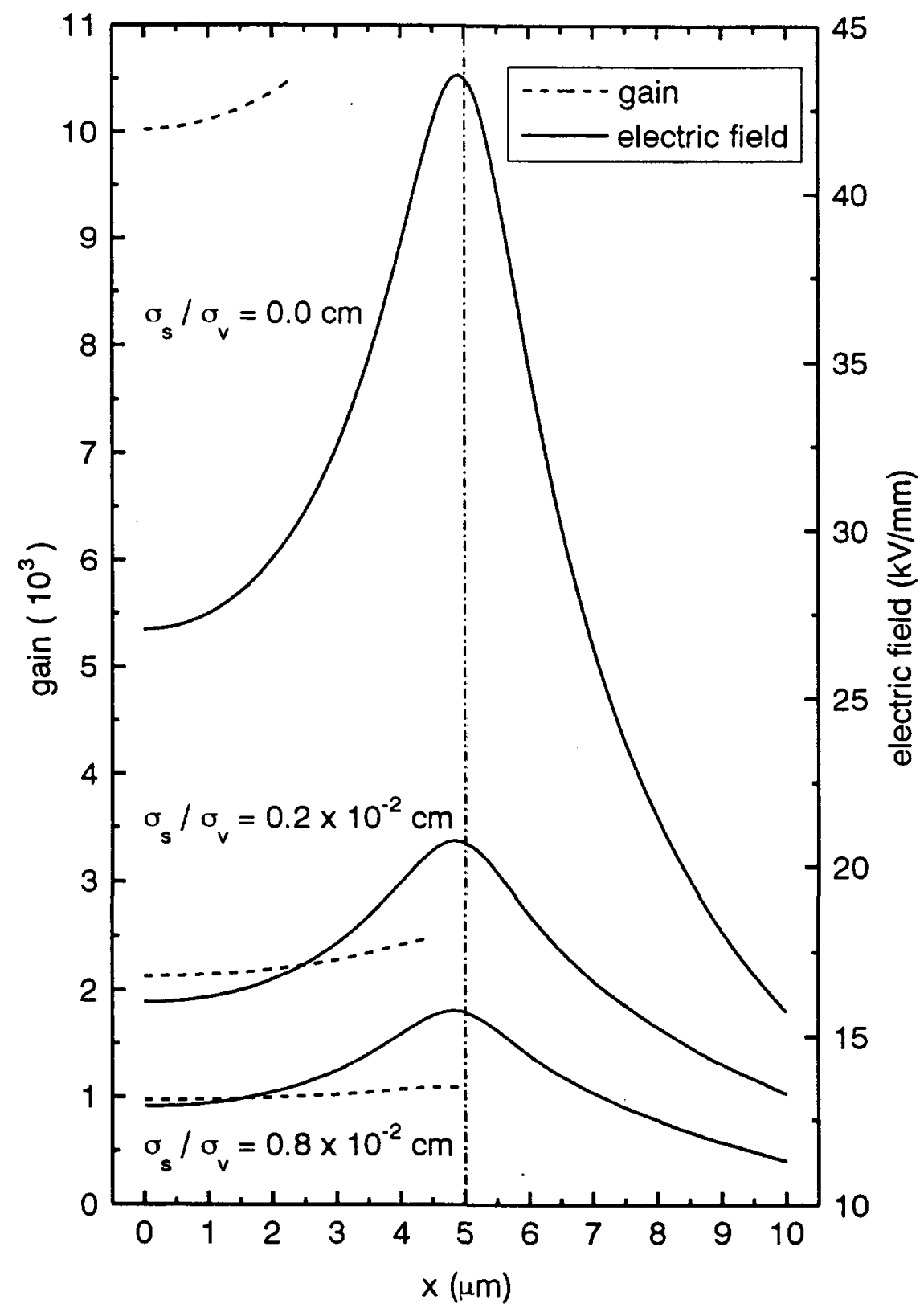

Figure 9: Electric field and gas amplification across the anode for different ratios of surface to bulk conductivity. The field is computed at a distance of $1 \mu \mathrm{m}$ from the anode plane. The dash-dotted line indicates the edge of the anode. 


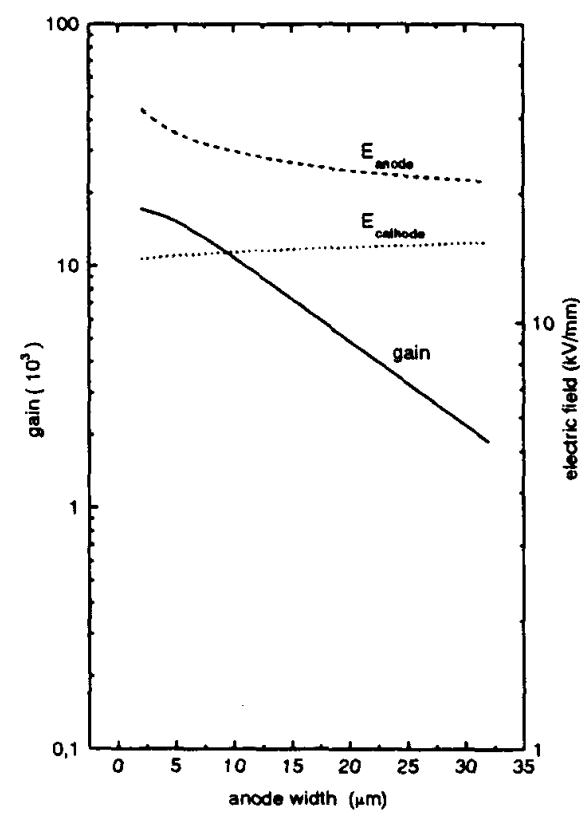

(a)

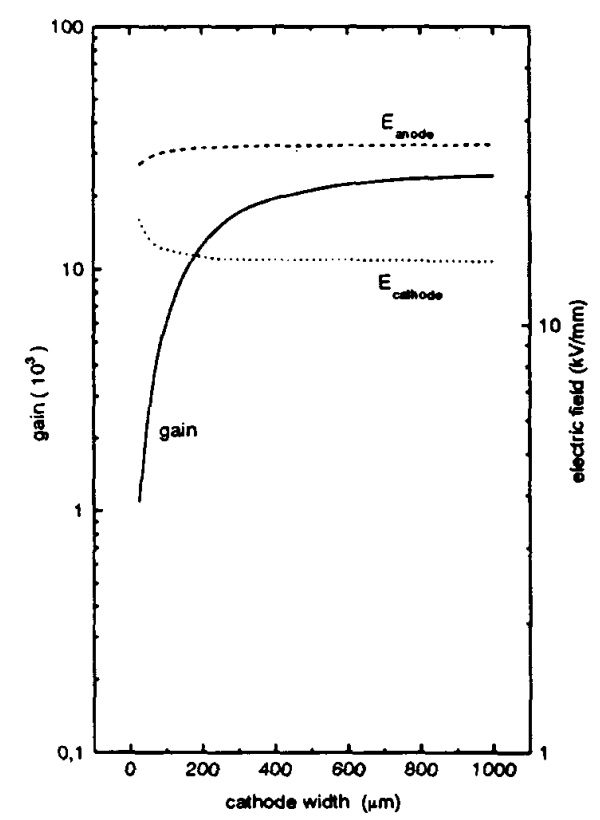

(b)
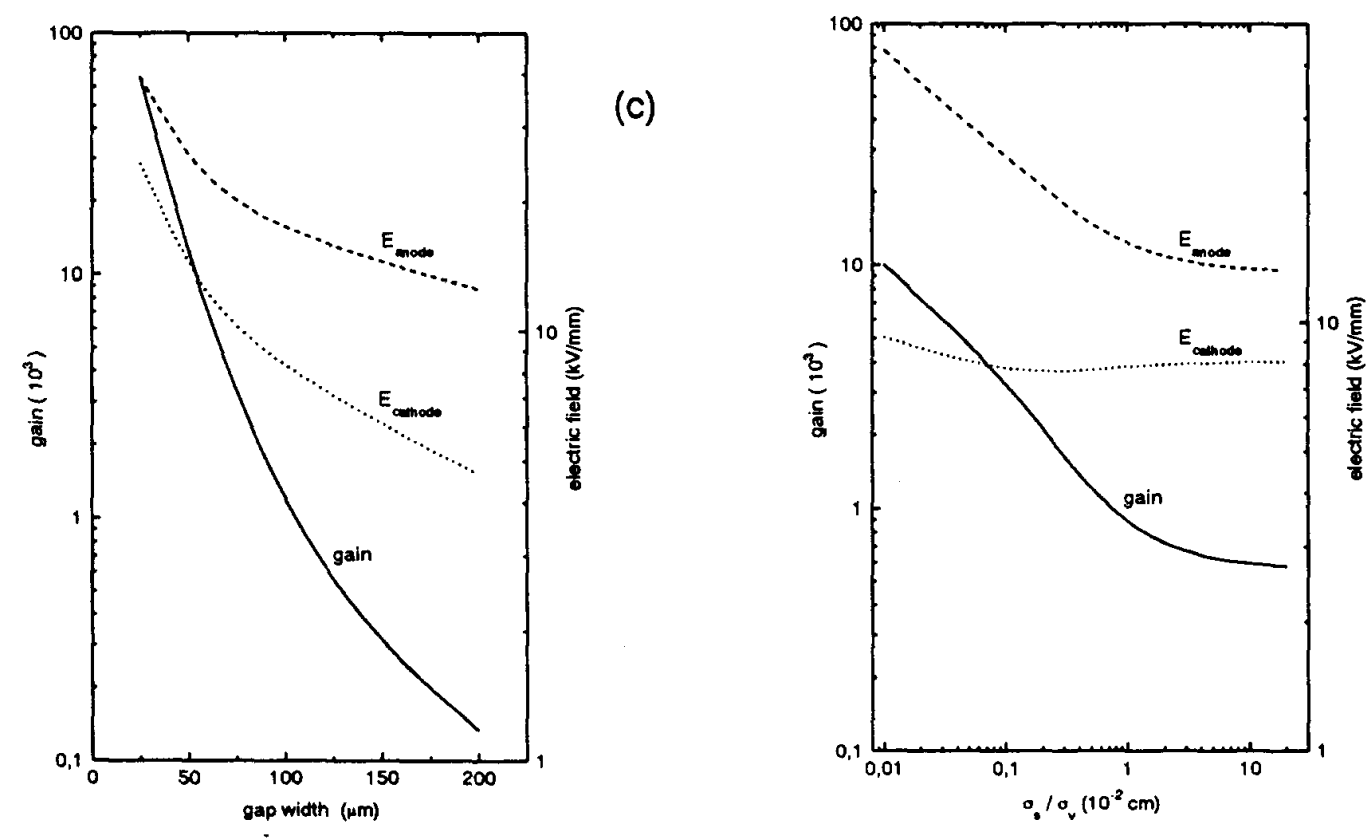

(d)

Figure 10: Gain and electric field at cathode edge and anode edge for different anode widths (a), cathode widths (b), gap widths (c) and for different ratios of surface to bulk conductivity (d) 


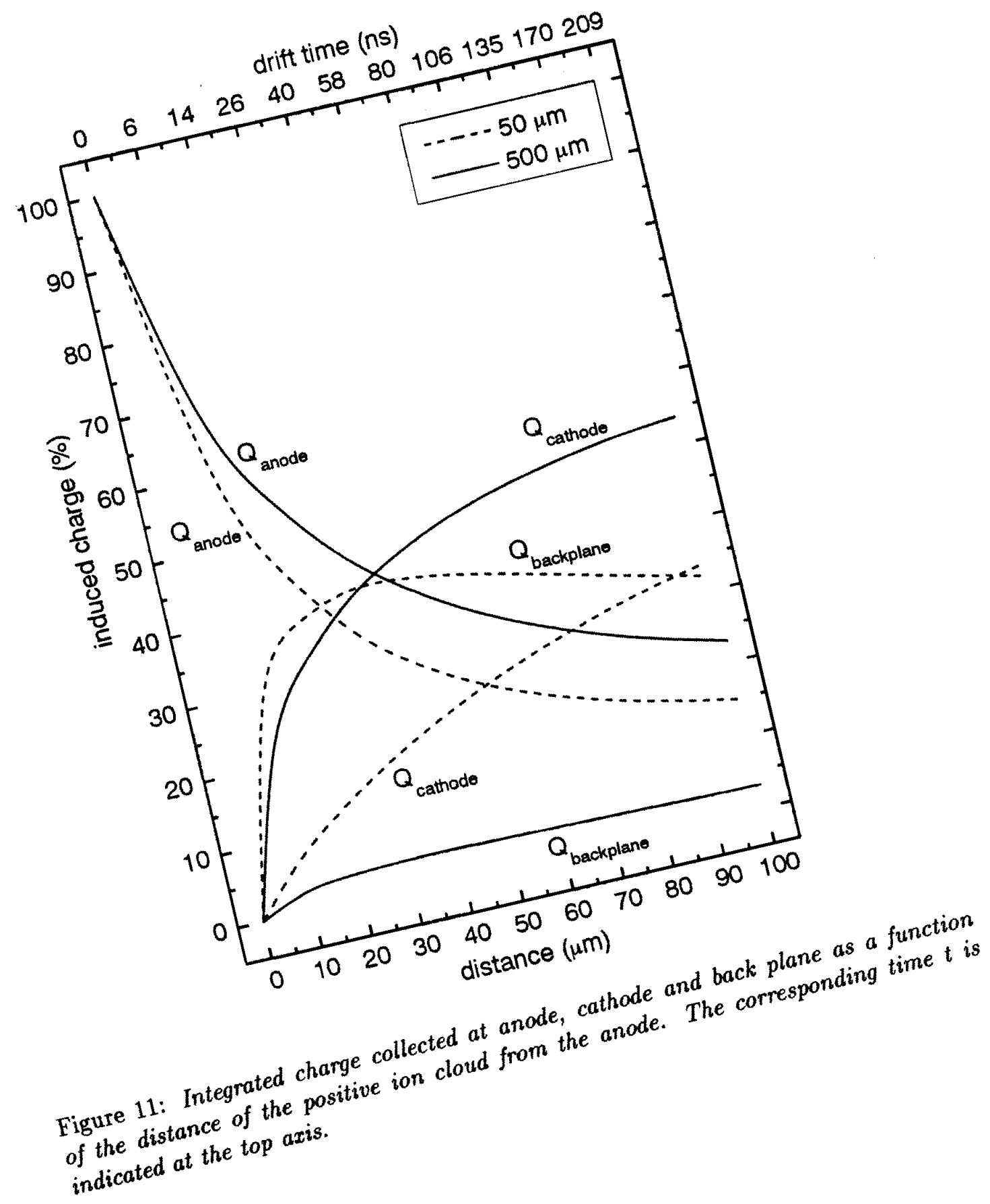


of the gain on $w_{C}$, which has also been observed experimentally [4]. The increase in gain is accompanied with a diminishing field at the cathode. The size of the cathode strips is limited in most applications by the acceptable cell width. For a jet-chamber like geometry, where the particles travel parallel to the substrates large cells are welcome in order to increase the primary ionization. In figure $10 \mathrm{~d}$ we display the variation of the gain and the field with the conductivity ratio $R_{\sigma}$. The gain decreases with increasing surface conductivity. This result suggests that low bulk resistivity substrates are favorable for high gas amplifications.

\subsection{Anode capacitances}

The electric signals obtained from MSGCs depend critically on the capacitances of the anode strip. We estimate this quantity, by applying a voltage to the anode strip and grounding the other electrodes. The capacitance scales with the dielectric constant of the substrate material, for which we chose a value of five in the following examples.

For some selected geometric parameters the anode capacitance and the couplings to the adjacent electrodes and to the back plane are given in table 2 . The anode capacitance lies typically in the range of 30 to $50 \mathrm{pF} / \mathrm{m}$. As expected, it increases with the width of the anode strips, decreases with the gap width and the thickness of the substrate and depends weakly on the width of the cathode strips. For thick substrates, $d_{\text {subst }}>200$ $\mu m$ the capacitances of the cathode strips dominate, for thin substrates mainly the back electrode contributes. The coupling to the adjacent anodes is sizeable for thick substrates and small pitches.

\subsection{Induced signals}

For some applications the signals induced on electrodes or pads by the moving cloud of the positive ions are of interest. The spatial precision can be improved using the difference of the signals induced in the two adjacent potential strips [20]. For synchrotron radiation detectors a pad readout from the back plane is foreseen. Our program allows to compute the induced signal on an infinitely long strip. The fraction of the anode signal which is induced on a square pad will be much smaller especially for thick substrates where the charge distribution is rather wide.

In figure 11 we show the charge induced by a test charge located above the center of the anode as a function of its distance from the anode. Substrates of thicknesses of 50 $\mu m$ and $500 \mu m$ were considered with the cell parameters $w_{A}=10 \mu m, w_{G}=120 \mu m$ and $w_{C}=200 \mu m$. The fraction of the test charge induced on the anode $Q_{\text {anode }}$ decreases to about twenty percent at $100 \mu m$ distance. Correspondingly the charge $Q_{\text {cath }}$ on the cathode strips increases.

The signal induced on the fraction of the back plane which covers the full cell is also shown in figure $11 . Q_{b a c k}$ rises quickly with the distance of the test charge from the anode, reaches a maximum at about $40 \mu \mathrm{m}$ and then decreases slowly. In figure 11 also the ion drift time is given. This allows to estimate the pulse shapes for a single ionization cluster. The voltages used in the simulation were $V_{A}=500 \mathrm{~V}, V_{D}=-3 \mathrm{kV}$ and $V_{B}=0$. The time scale is almost identical for the thick and the thin substrate.

In table 3 we summarize the induced signals for different geometric parameters for a $50 \mu m$ distant test charge. 


\begin{tabular}{|r|r|r|r|r|r|r|r|}
\hline \hline $\begin{array}{r}w_{\text {anode }} \\
(\mu \mathrm{m})\end{array}$ & $\begin{array}{r}w_{\text {gap }} \\
(\mu \mathrm{m})\end{array}$ & $\begin{array}{r}w_{\text {cath }} \\
(\mu \mathrm{m})\end{array}$ & $\begin{array}{r}d_{\text {subst }} \\
(\mu \mathrm{m})\end{array}$ & $\begin{array}{r}C_{\text {anode }} \\
(\mathrm{pF} / \mathrm{m})\end{array}$ & $\begin{array}{r}Q_{\text {cath }} \\
(\%)\end{array}$ & $\begin{array}{r}Q_{\text {adj }} \\
(\%)\end{array}$ & $\begin{array}{r}Q_{\text {back }} \\
(\%)\end{array}$ \\
\hline 10 & 120 & 200 & 1000 & 36 & 79 & 6 & 13 \\
10 & 120 & 200 & 500 & 36 & 73 & 4 & 22 \\
10 & 120 & 200 & 200 & 38 & 50 & 1 & 48 \\
10 & 120 & 200 & 100 & 42 & 24 & 1 & 75 \\
10 & 120 & 200 & 50 & 50 & 8 & 1 & 92 \\
\hline 10 & 120 & 100 & 500 & 35 & 68 & 5 & 24 \\
10 & 120 & 100 & 200 & 38 & 49 & 2 & 48 \\
10 & 120 & 100 & 100 & 42 & 24 & 1 & 73 \\
\hline 10 & 120 & 400 & 500 & 36 & 76 & 2 & 22 \\
10 & 120 & 400 & 200 & 38 & 51 & 1 & 48 \\
10 & 120 & 400 & 100 & 42 & 24 & 1 & 75 \\
\hline 10 & 50 & 200 & 500 & 44 & 84 & 4 & 11 \\
10 & 50 & 200 & 200 & 44 & 75 & 1 & 23 \\
10 & 50 & 200 & 100 & 46 & 56 & 1 & 43 \\
10 & 50 & 200 & 50 & 51 & 30 & 1 & 69 \\
\hline 10 & 50 & 100 & 1000 & 43 & 83 & 8 & 5 \\
10 & 50 & 100 & 500 & 43 & 80 & 6 & 12 \\
10 & 50 & 100 & 200 & 44 & 73 & 3 & 24 \\
10 & 50 & 100 & 100 & 46 & 56 & 1 & 43 \\
10 & 50 & 100 & 50 & 51 & 30 & 1 & 69 \\
\hline 10 & 200 & 200 & 500 & 35 & 61 & 3 & 35 \\
10 & 200 & 200 & 200 & 36 & 31 & 1 & 68 \\
10 & 200 & 200 & 100 & 41 & 10 & 1 & 89 \\
\hline 10 & 400 & 200 & 500 & 30 & 38 & 2 & 58 \\
10 & 400 & 200 & 200 & 35 & 10 & 1 & 88 \\
10 & 400 & 200 & 100 & 41 & 3 & 0 & 96 \\
\hline 5 & 120 & 200 & 500 & 31 & 72 & 4 & 23 \\
5 & 120 & 200 & 200 & 33 & 51 & 1 & 48 \\
\hline
\end{tabular}

Table 2: Anode capacitance and fraction of anode charge induced at the adjacent cathode strips $Q_{c a t h}$, the adjacent anodes $Q_{a d j}$ and the back plane $Q_{b a c k}$. 


\begin{tabular}{|r|r|r|r|r|r|r|r|}
\hline \hline $\begin{array}{r}w_{\text {anode }} \\
(\mu m)\end{array}$ & $\begin{array}{r}w_{\text {gap }} \\
(\mu m)\end{array}$ & $\begin{array}{r}w_{\text {cath }} \\
(\mu m)\end{array}$ & $\begin{array}{r}d_{\text {subst }} \\
(\mu m)\end{array}$ & $\begin{array}{r}Q_{\text {anode }} \\
(\%)\end{array}$ & $\begin{array}{r}Q_{\text {cath }} \\
(\%)\end{array}$ & $\begin{array}{r}Q_{\text {back }} \\
(\%)\end{array}$ & $\begin{array}{r}Q_{\text {adj }} \\
(\%)\end{array}$ \\
\hline \hline 10 & 120 & 200 & 500 & 36 & 47 & 4 & 2 \\
10 & 120 & 200 & 200 & 34 & 40 & 12 & 3 \\
10 & 120 & 200 & 100 & 31 & 32 & 25 & 3 \\
10 & 120 & 200 & 50 & 25 & 24 & 40 & 2 \\
\hline 10 & 120 & 100 & 500 & 36 & 42 & 4 & 2 \\
10 & 120 & 100 & 200 & 34 & 36 & 13 & 3 \\
10 & 120 & 100 & 100 & 31 & 28 & 25 & 3 \\
\hline 5 & 120 & 200 & 500 & 31 & 51 & 4 & 2 \\
5 & 120 & 200 & 200 & 30 & 43 & 14 & 3 \\
\hline 10 & 200 & 200 & 500 & 41 & 37 & 8 & 3 \\
10 & 200 & 200 & 200 & 38 & 27 & 24 & 3 \\
10 & 200 & 200 & 100 & 33 & 18 & 40 & 2 \\
10 & 200 & 100 & 500 & 41 & 33 & 8 & 3 \\
\hline 10 & 400 & 200 & 500 & 46 & 23 & 21 & 3 \\
10 & 400 & 200 & 200 & 40 & 11 & 42 & 2 \\
10 & 400 & 200 & 100 & 33 & 6 & 55 & 2 \\
10 & 400 & 100 & 500 & 46 & 20 & 21 & 3 \\
\hline \hline
\end{tabular}

Table 3: Charges induced by a test charge at the anode $\left(Q_{\text {anode }}\right)$, at the adjacent cathode strips $\left(Q_{\text {cath }}\right)$, at the adjacent anode strips $\left(Q_{a d j}\right)$ and at the back plane $\left(Q_{\text {back }}\right)$. The test charge is located $50 \mu \mathrm{m}$ above the anode.

As expected the width of the gap between the anode and the cathode strips has a sizeable effect on the amount of charge induced at the back plane whereas varying the width of the cathode or anode strips has little influence.

For the $500 \mu m$ thick substrates $Q_{\text {back }}$ is probably too small $(\approx 4 \%)$ to be useful. For square pads it would be further reduced drastically. An additional problem for the thick substrates is the relatively large signal $Q_{a d j}$ induced in the adjacent cell which is only by a factor two smaller. These problems can partially be reduced by choosing a large gap between anode and cathode strips.

The situation is much more favorable for thinner substrates, where up to $50 \%$ of the charge is collected in the back plane cell. Here also the charge distribution at the back plane is much narrower than for the thick substrates. This leads to acceptable losses when replacing the strips by pads. However, for very thin substrates one has to keep in mind that large gaps between anode and cathode strips are not compatible with a back plane electrode, except for very high surface conductivities. Field lines from either the anode or the drift electrode would end on the substrate surface. An acceptable solution could be a $100 \mu m$ thick substrate with a $400 \mu m$ wide gap.

For a thin suspended substrate and a pick-up electrode separated by an air gap the signal is even smaller than for a thick plate.

The asymmetry between the two cathode strips adjacent to the anode strip can be 


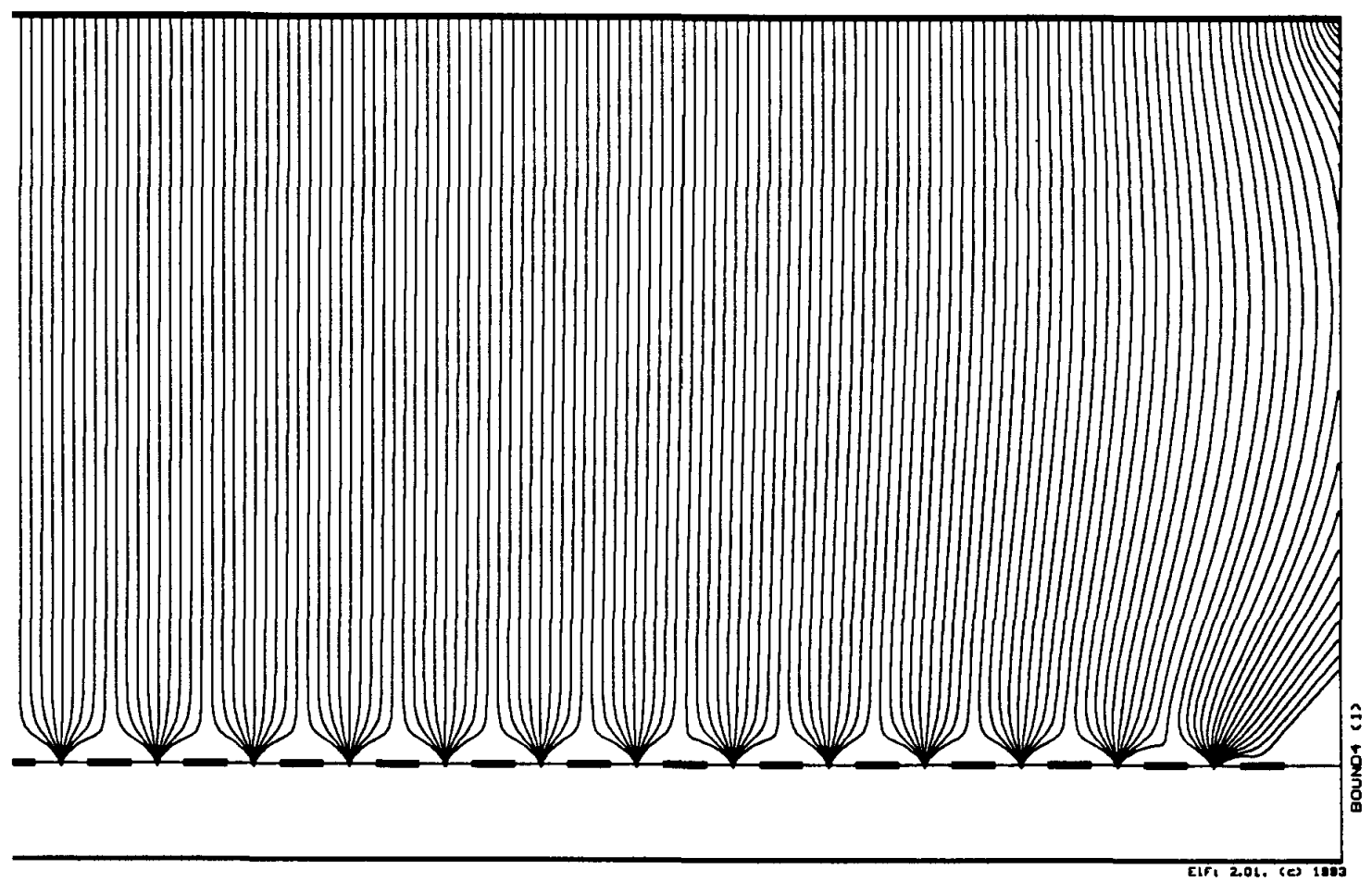

Figure 12: Electric field map near the border of the strip pattern.

used to improve the spatial resolution. Assuming that the positive ions travel back along the field line of the incoming primary electrons, maximum asymmetries of $30 \%$ to $50 \%$ can be expected. This value is not very sensitive to the geometry. However, when the anode is narrow and when device is operated at high gain the ion cloud can extend over the full anode and loose the spatial information contained in the primary ionisation.

\subsection{Border effects}

The spatial resolution of MSGCs depends on the sharing of the collected primary ionization by adjacent anodes. In the drift region the electric field is perpendicular to the substrate. However near the border noticeable distortions occur. This is shown in the field map of figure 12. An increased field strength is expected in the border gap. The fields at the edge of the outermost potential strip and the last anode strip are higher by only $3 \%$ compared to the values at the center of the chamber. This is a rather small effect which can be tolerated or avoided by a special design of the border strips.

\section{Summary and Conclusions}

We have simulated the electric field and the gas amplification in MSGCs and compared to measurements. From the simulation we conclude that the performance depends critically on the ratio of surface conductivity to bulk conductivity. High values of this ratio are desirable for stable operating conditions but require higher voltages. The gas 
amplification is constant across the anode. It can be increased significantly by using wide cathode strips. Cross talk to the adjacent anode is bellow $3 \%$ for most geometries and negligible for thin substrates with grounded back plane. The anode capacitance amounts to about 30 to $50 \mathrm{pF} / \mathrm{m}$. It varies little with the geometry of the electrodes and the substrate. Signals induced at the back plane are usually bellow $10 \%$ of the anode signal for thick $(>200 \mu \mathrm{m})$ substrates with narrow $(<100 \mu \mathrm{m})$ gaps between anode and cathode strips. They can reach $50 \%$ for $100 \mu m$ thin substrates and $400 \mu m$ wide gaps. The asymmetry of the signals induced at the cathode strips should allow to apply the induction principle to improve the spatial resolution.

\section{Acknowledgement}

We thank J. Grosch for assistance in the measurements and Dr. H.-J. Besch for many helpful comments.

\section{References}

[1] A. Oed, Nucl. Instr. and Meth. A263 (1988) 351.

[2] R. Bouclier et al. Nucl. Instr. and Meth. A332 (1993) 100.

[3] F. Angelini et al., Pisa preprint INFN PI/AE 93/10 (1993).

[4] S. Schmidt et al., to appear in Nucl. Instr. and Meth. A (1993)

[5] J.J. Florent et al., CERN-PPE 92-78 (1992).

[6] Schmitz, NIKHEF-PPE 93-07 (1992).

[7] S. Schmidt et al., Presentation at the Workshop on Gaseous Microstrip Proportional Chambers, Grenoble (1993), unpublished.

[8] F. Angelini et al., Presentation at the Workshop on Gaseous Microstrip Proportional Chambers, Grenoble (1993), unpublished.

[9] L. Zagar et al, Glastechnische Berichte 48 (1975) 99

[10] H. Schiller, Ann. d. Phys. 81 (1926) 32

[11] G. Schulze, Ann. d. Phys. 37 (1912) 435

[12] J.E. Bateman et al. Rutherford Appleton Lab. preprint, RAL-92-085

[13] R. Bouclier et al. Nucl. Instr. and Meth. A323 (1992) 240

[14] W. Weihs et al., Nucl. Instr. and Meth. A281 (1989) 393.

[15] M.W. Charles, Jour. of Phys. E5 (1972) 95

[16] J.E. Bateman, Rutherford Appleton Lab. preprint, RAL-93-019

[17] H. Miyahara et al., Nucl. Instr. and Meth. A241 (1985) 186 
[18] R. G. Filho et al, IEEE Trans. on Electr. Insul. E1-21,3 (1986)

[19] T. Nagae et al., Nucl. Instr. and Meth. A323 (1992) 236

[20] E. Roderburg et al., Nucl. Instr. and Meth. A252 (1986) 285

[21] F. Angelini et al., Nucl. Instr. and Meth. A283 (1989) 755 
